\title{
Endosulfan Poisoning Resulting from Skin Exposure
}

\author{
Atul Jindal • Naveen Sankhyan
}

Received: 14 July 2011 / Accepted: 25 November 2011 /Published online: 10 December 2011

(C) Dr. K C Chaudhuri Foundation 2011

Sir,

In the letter to the editor by Kamate $\mathrm{M}$ et al., [1] important aspects of neurotoxicity of endosulfan have been highlighted. We wish to share our experience in handling a child with endosulfan poisoning resulting from skin exposure. We also want to add a few specific points in the management.

A 2-y-old girl presented to the emergency with history of continuous generalized tonic clonic seizure for the past $1 \mathrm{~h}$. There was a history of endosulfan application on head $2 \mathrm{~h}$ prior to the symptoms. The mother had applied endosulfan to remove head lice. There was no other significant history or any significant systemic findings. The seizure was immediately aborted by intravenous diazepam and child was loaded with intravenous phenytoin. The child's head was shaved and surface decontamination was done with soap and water. The child persisted to be irritable for the next $12 \mathrm{~h}$ but there was no recurrence of seizures in the hospital. Her hemoglobin was $8.6 \mathrm{~g} / \mathrm{dL}$, and the total leukocyte count was $10,600 / \mathrm{mm}^{3}$. Her serum electrolytes, blood gases, liver and renal functions were normal. She was asymptomatic and discharged on day 3 of presentation. Parents were counseled regarding the safe handling of endosulfan and other pesticides at home.

Endosulfan and other organochlorines are central nervous system stimulants. They are thought to exert their toxic effects through inhibition of $\gamma$ amino butyric acid (GABA) receptors. Toxicity commonly manifests as altered sensorium

\footnotetext{
A. Jindal

Division of Emergency Medicine and Intensive Care, Department of Pediatrics, Advanced Pediatric Center, Post Graduate Institute of Medical Education and Research, Chandigarh, India

N. Sankhyan $(\bowtie)$

Department of Pediatrics, Advanced Pediatric Center, Post Graduate Institute of Medical Education and Research, Chandigarh 160012, India

e-mail:drnsankhyan@yahoo.co.in
}

(81\%), generalised seizures (75\%) including status epilepticus (33\%) [2]. Control of seizures is an important step in management of this toxicity. There are no trials investigating an optimal anti-convulsant or treatment algorithm for drug or toxin induced seizures. In general, benzodiazepines followed by barbiturates are the first and second-line therapies for drug or toxin induced seizures unless a specific antidote is available $[3,4]$. Benzodiazepines and barbiturates are preferred over phenytoin because both these anticonvulsants are agonists at the GABA-A receptors, which facilitate chloride influx through the chloride ionophore, enhancing GABA inhibition [5]. Although phenytoin is the second line agent indicated in the treatment of most causes of status epilepticus, it is not as useful in the management of drug or toxin induced seizures. Moreover its use can potentially enhance cardiac toxicity of certain toxins. There is general lack of awareness to this fact, which is evident by the emergency room use of phenytoin in our patient and the one reported by authors.

While using benzodiazapines and/or barbiturates it is important to watch for respiratory depression and hemodynamic status. If facilities for respiratory or cardiovascular support are not readily available then alternative antiepileptics like valproate or levetiracetam may be safer options.

\section{References}

1. Kamate M, Jain A. Accidental endosulfan ingestion in a toddler. Indian J Pediatr. 2011;78:884-5.

2. Moses V, Peter JV. Acute intentional toxicity: endosulfan and other Organochlorines. Clin Toxicol. 2010;48:539-44.

3. Wills B, Erickson T. Drug and toxin-associated seizures. Med Clin North Am. 2005;89:1297-321.

4. Wiegand T. Phenytoin is not successful in preventing cocaineinduced seizures: a response to the article, "Cocaine body packing in pregnancy". Ann Emerg Med. 2007;49:543-4.

5. Shannon M, McElroy EA, Liebelt EL. Toxic seizures in children: case scenarios and treatment strategies. Pediatr Emerg Care. 2003;19:206-10. 